\title{
BAG3-mediated Mcl-1 stabilization contributes to drug resistance via interaction with USP9X in ovarian cancer
}

\author{
SHUTARO HABATA, MASAHIRO IWASAKI, ASUKA SUGIO, MIWA SUZUKI, MASATO TAMATE, \\ SEIRO SATOHISA, RYOICHI TANAKA and TSUYOSHI SAITO
}

Department of Obstetrics and Gynecology, Sapporo Medical University, Sapporo 060-8543, Japan

Received January 21, 2016; Accepted March 30, 2016

DOI: 10.3892/ijo.2016.3494

\begin{abstract}
Paclitaxel in combination with carboplatin improves survival among patients with susceptible ovarian cancers, but no strategy has been established against resistant ovarian cancers. BAG3 (Bcl-2-associated athanogene 3) is one of six BAG family proteins, which are involved in such cellular processes as proliferation, migration and apoptosis. In addition, expression of BAG3 with Mcl-1, a Bcl-2 family protein, reportedly associates with resistance to chemotherapy. Our aim in this study was to evaluate the functional role of BAG3 and Mcl-1 in ovarian cancer chemoresistance and explore possible new targets for treatment. We found that combined expression of BAG3 and Mcl-1 was significantly associated with a poor prognosis in ovarian cancer patients. In vitro, BAG3 knockdown in ES2 clear ovarian cancer cells significantly increased the efficacy of paclitaxel in combination with the Mcl-1 antagonist MIM1, with or without the Bcl-2 family antagonist ABT737. Moreover, BAG3 was found to positively regulate Mcl-1 levels by binding to and inhibiting USP9X. Our data show that BAG3 and Mcl-1 are key mediators of resistance to chemotherapy in ovarian cancer. In BAG3 knockdown ES2 clear ovarian cancer cells, combination with ABT737 and MIM1 enhanced the efficacy of paclitaxel. These results suggest that inhibiting BAG3 in addition to antiapoptotic Bcl-2 family proteins may be a useful therapeutic strategy for the treatment of chemoresistant ovarian cancers.
\end{abstract}

\section{Introduction}

Clear cell adenocarcinoma (CCC) of the ovary accounts for $10 \%$ of epithelial ovarian cancers and is an entity distinct from

Correspondence to: Dr Masahiro Iwasaki, Department of Obstetrics and Gynecology, Sapporo Medical University, South-1, West-16, Chuo-ku, Sapporo, Hokkaido 060-8543, Japan

E-mail: miwasaki@sapmed.ac.jp

Abbreviations: BAG3, Bcl-2-associated athanogene 3; CCC, clear cell adenocarcinoma; XTT, 2, 3-bis-(2-methoxy-4-nitro-5-sulfophenyl)$2 H$-tetrazolium-5-carboxanilide; PTX, paclitaxel; PFS, progressionfree survival; shRNA, short hairpin RNA; IP, immunoprecipitation

Key words: BAG3, Mcl-1, ovarian cancer, apoptosis, paclitaxel, chemoresistance other ovarian carcinomas. For example, CCC exhibits a more chemoresistant phenotype than other histological types, which leads to a poorer prognosis. Although the combination of carboplatin and paclitaxel has been established as the standard therapy for ovarian cancer (1), with serous and endometrioid adenocarcinomas responding well to this regimen, $\mathrm{CCC}$ shows lower response rates $(2,3)$. Moreover, the incidence of CCC has been increasing; it is now estimated to account for $20 \%$ of ovarian cancers in Japan and $~ 5-6 \%$ in Europe (3). New target-based therapies for the treatment of CCC remains an unmet need in these patients.

Apoptotic cell death is critical for the maintenance of tissue homeostasis in healthy organisms, providing an efficient and safe mechanism to remove unwanted cells. Impairment of apoptosis is a crucial step in tumor development (4) and renders tumor cells more resistant to conventional cytotoxic therapy (5). The key regulators of apoptosis are interacting proteins of the Bcl-2 family, which has been subdivided into three subfamilies: a pro-survival subfamily $(\mathrm{Bcl} 2$ and the closely related proteins $\mathrm{Bcl}-\mathrm{X}_{\mathrm{L}}, \mathrm{Bcl}_{-\mathrm{w}}$ and $\left.\mathrm{Mcl}-1\right)$ and two pro-apoptotic subfamilies that include the initiator BH3-only proteins (Bim, PUMA, BAD and NOXA) and the cell death mediators (BAX and BAK) (6,7). The interactions among these intracellular proteins determine whether a cell lives or dies, and alterations in their expression and function are associated with cancer development $(8,9)$. ABT737 is a smallmolecule inhibitor of the anti-apoptotic proteins $\mathrm{Bcl}-2, \mathrm{Bcl}-\mathrm{X}_{\mathrm{L}}$ and $\mathrm{Bcl}_{-}$, which shows single-agent activity and increases sensitivity to chemotherapeutic agents (10). However, cells expressing high levels of Mcl-1 show resistance to ABT737 (11). Mcl-1 is also thought to be a crucial pro-survival factor responsible for resistance to antitublin agents such as paclitaxel (12). Therefore, inhibiting Mcl-1, as well as Bcl-2, Bcl- $\mathrm{X}_{\mathrm{L}}$ and $\mathrm{Bcl}_{-}$, may be required to overcome ovarian cancer chemoresistance. Recently, Mcl-1 inhibitor molecule 1 (MIM1), a selective small-molecule inhibitor of Mcl-1 $\left(\mathrm{IC}_{50}, 4.8 \mu \mathrm{M}\right)$ that overcomes Mcl-1-dependent leukemia cell survival, was reported (13). MIM1 selectively affects Mcl-1, with no effect on Bcl-2 or Bcl- $\mathrm{X}_{\mathrm{L}}$. In this report we investigated the effect of combining paclitaxel with ABT-737 or/and MIM1 on ovarian cancer cell viability.

Bcl2-associated athanogene 3 (BAG3) is one of six BAG family proteins. Via its BAG domain, BAG3 interacts with and regulates the folding of Hsc70/Hsp70 (14,15). In addition 
to the BAG domain, BAG3 contains a WW domain near its $\mathrm{N}$-terminus and a proline-rich region (multiple PXXP motifs) $(16,17)$, and our earlier study showed that BAG3 also regulates cell motility and tumor growth, invasion and metastasis (18). Moreover, several lines of evidence indicate that downregulation of BAG3 enhances chemotherapy-mediated apoptosis among cancer cells (19-21), which is consistent with the recent finding that BAG3 stabilizes Mcl-1, Bcl-2 and Bcl- $\mathrm{X}_{\mathrm{L}}$, thereby promoting cancer cell survival $(22,23)$. The precise mechanism by which BAG3 exerts these effects remains unclear. It is known, however, that the deubiquitinase USP9X (ubiquitinspecific peptidase $9, \mathrm{X}$-linked) is among the proteins that co-immunoprecipitate with Mcl-1 (24), and that removing the poly-ubiquitin chains from Mcl-1 stabilizes it and confers resistance to apoptosis. In this manner, USP9X reportedly promotes tumor cell survival (25).

In this study, we investigated the mechanism by which BAG3 confers chemoresistance to ovarian cancer cells. We found that BAG3 stabilizes Mcl-1 by interacting with USP9X. We previously showed that BAG3 expression relates to clinical severity and prognosis in ovarian cancer patients (26). Here we assessed Mcl-1 expression in ovarian cancer tissues and its correlation with the clinical state, and evaluated the effect of ABT-737 and MIM1 on ovarian cancer cell sensitivity to paclitaxel.

\section{Materials and methods}

Cells and cell culture. Two established ovarian cancer cell lines were used in this study. The AMOC2 line was established from a serous adenocarcinoma, while the ES2 line was established from a clear cell carcinoma. AMOC2 cells were cultured in RPMI-1640 (Gibco, Grand Island, NY, USA) supplemented with $10 \%$ fetal bovine serum (Hyclone, Logan, UT, USA) and $1 \%$ penicillin/streptomycin (Gibco). ES2 cells were cultured in Dulbecco's modified Eagle's medium (DMEM) (Gibco) supplemented with $10 \%$ FBS and $1 \%$ penicillin/streptomycin. Both cell lines were maintained in a $\mathrm{CO}_{2}$ incubator $\left(5 \% \mathrm{CO}_{2}\right)$ at $37^{\circ} \mathrm{C}$.

BAG3 overexpression. AMOC2 cells were transfected with the expression vector pcDNA-BAG3, encoding fulllength BAG3, or with empty pcDNA vector (control) using Lipofectamine 3000 regent (Invitrogen, Carlsbad, CA, USA) according to the manufacturer's protocol. After $24 \mathrm{~h}$, the cells were split and allowed to adhere overnight.

Real-time quantitative reverse transcription PCR ( $q R T-P C R)$ for microRNA. Total RNA was extracted from cells using TRIzol regent (Invitrogen), after which reverse transcription was performed with $10 \mu \mathrm{g}$ of total RNA using a TaqMan ${ }^{\circledR}$ MicroRNA Reverse Transcription kit (Applied Biosystems, Foster, CA, USA) and sequence-specific RT primers from the TaqMan MicroRNA assays (Applied Biosystems) according to the manufacturer's instructions. Separate reverse transcription reactions were run for each TaqMan MicroRNA assay on every RNA sample. qRT-PCR was performed with cDNA using inventoried TaqMan MicroRNA assays and TaqMan Universal Master Mix II (Applied Biosystems). The assay was performed in triplicate, and the PCR amplification was performed using a
StepOnePlus $^{\text {TM }}$ Real-Time PCR system (Applied Biosystems). Gene expression was calculated using the $2-\Delta \mathrm{Ct}$ method.

Mature miRNA transfection. Using Lipofectamine RNAiMAX (Invitrogen), cells grown in 6-cm dishes were transfected with miRNA-29b mirVana miRNA mimic (Ambion, Austin, TX, USA) to augment miR-29b activity, or with an inactive negative control for miRNA-29b mirVana miRNA mimic (Ambion). Alternatively, they were transfected with miRNA-29b mirVana miRNA inhibitor (Ambion) to diminish miR-29b activity or with a negative control for miRNA-29b mirVana miRNA inhibitor (Ambion).

Gene silencing using a short hairpin RNA (shRNA) vector. A gene silencing vector (pLTRH1) containing RNA polymerase promoter producing shRNA specific for BAG3 was used to transfect ES2 and AMOC2 cells. Oligonucleotides [5'-GAT CCCGTACCTGATGATCGAAGAGTTTCAAGAGAACTCT TCGATCATCAGGTATTTTTGGAG-3' (sense) and 5'-TCGA CTTCCAAAAAATACCTGATGATCGAAGAGTTCTCTT GAAACTCTTCGATCATCAGGTACGG-3' (antisense)] specific for mouse bag3 were synthesized and subcloned into the BglII and SalI sites, downstream of the H1 promoter (27). G3T-hi amphotrophic packaging cells (Takara Bio, Shiga, Japan) were transfected with pLTRH1bag3 puro or empty pLTRH1 puro vector according to the manufacturer's instructions to obtain a retroviral supernatant, which was added at a 1:5 ratio to DMEM or RPMI-1640 supplemented with $10 \%$ FBS and then used to infect ES2 or AMOC2 cells. Infected cells were then selected by incubation in medium containing $1.0 \mu \mathrm{g} / \mathrm{ml}$ puromycin (Gibco) for $48 \mathrm{~h}$ after infection. High-responder clones to BAG3 knockdown were selected for subsequent experiments.

siRNA transfection. Cells were transfected with Mcl-1 small interfering RNA (siRNA) (\#6315; Cell Signaling, MA, USA), control siRNA (\#6568; Cell Signaling), USP9X siRNA (SR305407; OriGene, MD, USA) or control siRNA (SR300004; OriGene) by Lipofectamine RNAiMAX (Invitrogen), and lysates were prepared $48 \mathrm{~h}$ after transfection.

Lysate production. Cell lysates were produced from subconfluent cell cultures. After scraping the cells from dishes, they were placed in RIPA buffer [50 mM Tris- $\mathrm{HCl}(\mathrm{pH} 8.0)$, $150 \mathrm{mM} \mathrm{NaCl}, 0.1 \% \mathrm{SDS}, 1 \% \mathrm{NP} 40$ and $0.5 \%$ sodium deoxycholate] containing a protease inhibitor cocktail (1:100 dilution; Thermo Scientific, Rockford, IL, USA). The cells were then lysed by sonication, after which the lysates were centrifuged at $15,000 \mathrm{rpm}$ for $15 \mathrm{~min}$ at $4^{\circ} \mathrm{C}$ to pellet the nuclei. The supernatant was then collected as the cell lysate.

Western blotting. After measuring the protein content, lysates was diluted in $2 \mathrm{X}$ sample buffer $[0.5 \mathrm{M}$ Tris- $\mathrm{HCl}(\mathrm{pH} 6.8)$, $10 \%$ SDS, $\beta$-mercaptoethanol and $1 \%$ BPB] and boiled for $5 \mathrm{~min}$ at $100^{\circ} \mathrm{C}$. Samples containing $20 \mu \mathrm{g}$ of protein were then electrophoresed ( $200 \mathrm{~V}$ for $35 \mathrm{~min}$ ) on SDS polyacrylamide gel, and the separated proteins were transferred onto PVDF membranes. After blocking with 5\% non-fat dry milk in TBS [10 mM sodium phosphate (pH 7.8), $150 \mathrm{mM} \mathrm{NaCl}$ and $0.05 \%$ Tween-20], the membrane was probed with the 
following primary antibodies: anti-BAG3 (1:1,000 dilution; gift of Dr S. Takayama), anti-Mcl-1 (1:500 dilution; S-19; Santa Cruz Biotechnology, Inc., Dallas, TX, USA), anti-Bcl- $X_{\mathrm{L}}$ (1:1,000 dilution; \#2762; Cell Signaling), anti-PARP (1:1,000 dilution; \#9542; Cell Signaling), anti-USP9X (1:5,000 dilution; ab99343, Abcam, Cambridge, UK) and anti- $\beta$-actin (1:5,000 dilution; A5441; Sigma-Aldrich, St. Louis, MO, USA). The protein was visualized using ECL Prime Western Blotting Detection Reagent and ImageQuant LAS 500 (GE Healthcare, Buckinghamshire, UK).

Cell viability assay. To test the responsiveness of the cells to paclitaxel under various culture conditions, cells were plated in $5 \%$ serum-containing medium in 96 -well plates $(5,000$ cells/ well) and incubated at $37^{\circ} \mathrm{C}$ under a $5 \% \mathrm{CO}_{2}$ atmosphere. After $24 \mathrm{~h}$, the medium was replaced with medium containing the indicated concentration of paclitaxel (Nippon Kayaku Co., Ltd., Tokyo, Japan), MIM1 (Merck Millipore, Darmstadt, Germany), ABT737 (Selleck Chemicals, Houston, TX, USA) or a combination of the three. Cell viability assays were then performed after $48 \mathrm{~h}$ using a Cell Proliferation Kit II (XTT; Roche Diagnostics, Mannheim, Germany). After the desired incubation period, $50 \mu 1$ of XTT labeling mixture was added to each well, and the cells were incubated for an additional $4 \mathrm{~h}$, after which absorbance at $492 \mathrm{~nm}$ was recorded using an ELISA plate reader.

Co-immunoprecipitation. Cell lysates were prepared in immunoprecipitation (IP) lysis buffer $(25 \mathrm{mM}$ Tris, $150 \mathrm{mM}$ $\mathrm{NaCl}, 1 \mathrm{mM}$ EDTA, $1 \%$ Nonidet P-40, 5\% glycerol and protease inhibitor cocktail (1:100 dilution, $\mathrm{pH}$ 7.4; Thermo Scientific) and clarified by centrifugation (14,000 x g, $20 \mathrm{~min})$. Anti-USP9X (Abcam) and anti-Myc-tag mAb (1:100 dilution; M192-3; MBL, Co., Ltd., Nagoya, Japan) were used for immunoprecipitation. Dynabeads Protein G (Life Technologies, Carlsbad, CA, USA) were incubated with antibody for $30 \mathrm{~min}$ at $4^{\circ} \mathrm{C}$. After washing beads with IP buffer, the beads with the immobilized antibody were incubated with lysates for $30 \mathrm{~min}$ at $4^{\circ} \mathrm{C}$. The beads were then washed three times with IP buffer, and the complexes were eluted in 2X SDS sample buffer $\left(5 \mathrm{~min}\right.$ at $\left.100^{\circ} \mathrm{C}\right)$, resolved by SDS-PAGE and analyzed by western blotting.

Patients for clinical and pathological analysis. Primary cancer tissue specimens were obtained from 51 patients operated on for ovarian cancer at Sapporo Medical University Hospital. All samples were preserved at $-80^{\circ} \mathrm{C}$ until used. In addition, the patient's clinical and pathological data were obtained from their medical records. None of the participating patients received preoperative treatment (e.g., neoadjuvant chemotherapy). All patients signed a consent form to participate.

Statistical analysis. Student's t-tests were used for statistical evaluation of the data. The Kaplan-Meier product limit method was used to compare progression-free survival among the patients, and the data obtained were evaluated using the log-rank test. Values of $\mathrm{P}<0.05$ were considered significant. SPSS 22.0 (IBM, Armonk, NY, USA) was used in analysis.
Table I. Mcl-1 expression and clinicopathological features in ovarian cancer.

\begin{tabular}{lcc}
\hline Characteristics & Value & $\begin{array}{c}\text { Mcl-1 positive } \\
\text { N }(\%)\end{array}$ \\
\hline Number & 51 & $11(21.6)$ \\
Age (mean, range) & $52,19-82$ & \\
Histology & & \\
Serous & 12 & $4(33.3)$ \\
Endometrioid & 17 & $5(29.4)$ \\
Clear & 10 & $1(10)$ \\
Mucinous & 6 & $1(16.6)$ \\
Others & 6 & $0(0)$ \\
Stage & & $1(5.8)$ \\
I & 17 & $0(0)$ \\
II & 2 & $9(32.1)$ \\
III & 28 & $1(25)$ \\
IV & 4 & \\
\hline
\end{tabular}

N, No. of cases; serous, serous adenocarcinoma; endometrioid, endometrioid adenocarcinoma; clear, clear cell carcinoma; mucinous, mucinous adenocarcinoma.

\section{Results}

Mcl-1 expression in primary ovarian cancer tissue. We previously found that BAG3 is associated with significantly higher risks of cancer progression and relapse (26), and that BAG3 upregulates Mcl-1 and mediates chemoresistance in ovarian cancer cells (28). In this study, we first investigated the expression of Mcl-1 in ovarian cancer patients. Lysates were prepared from tissue samples collected from 51 ovarian cancer patients and analyzed by immunoblotting. The patient characteristics are shown in Table I. Mcl-1 expression was detected in $11(21.6 \%)$ of the 51 samples. Upon application of FIGO stage classification, we found that the rate of Mcl-1 positivity was higher at stages III $(9 / 28,32.1 \%)$ and IV $(1 / 4,25 \%)$ than at stages I $(1 / 17,5.8 \%)$ and II $(0 / 2,0 \%)$, but there was no significant difference in Mcl-1 expression among FIGO stages, and no significant correlation between Mcl-1 expression and histologic type. Nonetheless, Kaplan-Meier analysis indicated that the progression-free survival (PFS) was poorer among Mcl-1-positive patients than Mcl-1-negative patients (median duration of PFS: 512 vs. 2,020 days, $\mathrm{P}=0.04$; Fig. 1A).

To investigate the correlation between BAG3 and Mcl-1 expression in ovarian cancer patients, we classified BAG3positive patients as being only BAG3-positive (Mcl-1 negative) or both BAG3- and Mcl-1-positive. Of the 34 BAG3-positive patients, Mcl-1 expression was detected in 10. Kaplan-Meier analysis showed that the PFS of patients in the BAG3- and Mcl-1-positive group was significantly poorer than in the only BAG3-positive group ( $\mathrm{P}=0.0301$; Fig. 1B). The median PFS in the BAG3- and Mcl-1-positive group was 440 days. By contrast, the only BAG3-positive group has not yet reached a median PFS time in our analysis. Moreover, Kaplan-Meier 
A

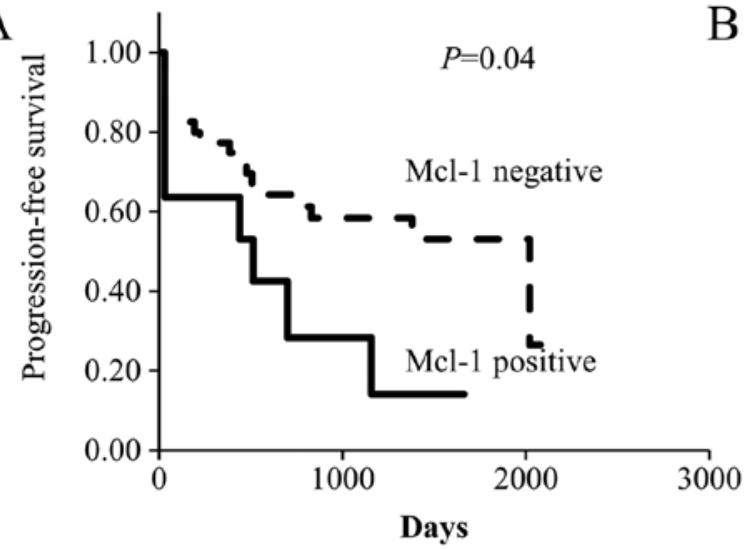

$\mathrm{B}$

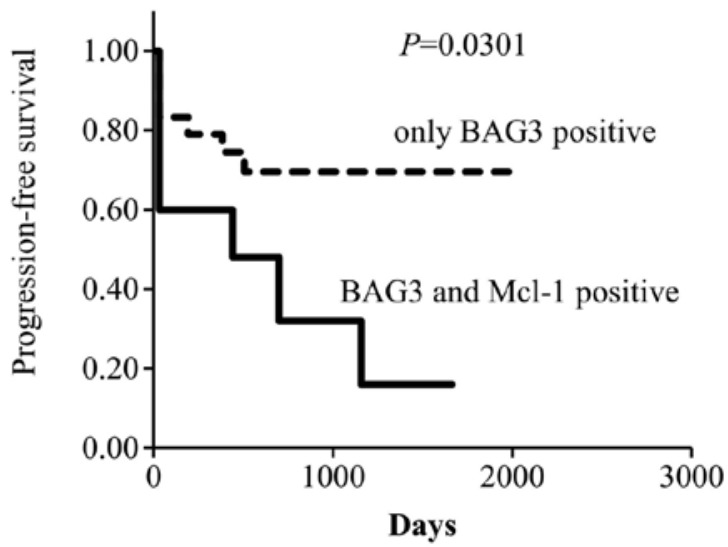

C

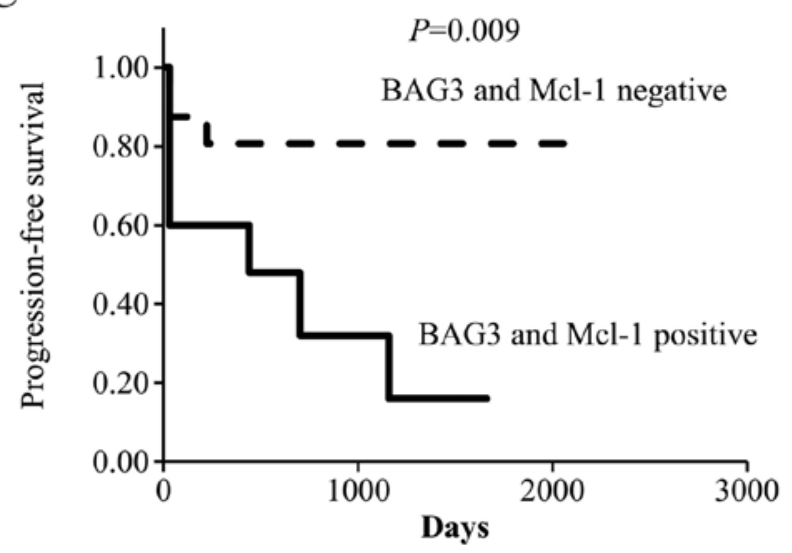

Figure 1. Effect of Mcl-1 and/or BAG3 expression on progression-free survival in ovarian cancer patients. (A) Kaplan-Meier ovarian cancer progression-free survival curves for Mcl-1-positive and Mcl-1-negative patients. Patients whose cancers expressed Mcl-1 showed significantly poorer progression-free survival ( $\mathrm{P}=0.04$; log-rank test). (B) Kaplan-Meier ovarian cancer progression-free survival curves for patients positive for both BAG3 and Mcl-1 and those positive for only BAG3. Patients whose cancers expressed both BAG3 and Mcl-1 also showed significantly poorer progression-free survival (P=0.03; log-rank test). (C) Kaplan-Meier ovarian cancer progression-free survival curves for patients positive for both BAG3 and Mcl-1 and those negative for both BAG3 and Mcl-1. Patients whose cancers expressed both BAG3 and Mcl-1 also showed significantly poorer progression-free survival (P=0.009; log-rank test).

analysis showed that the PFS of patients in the BAG3- and Mcl-1-positive group was significantly poorer than in the BAG3- and Mcl-1-negative group ( $\mathrm{P}=0.009$; Fig. 1C). Because only Mcl-1-positive patients (BAG3 negative) in total were 2 cases, we could not compare only Mcl-1-positive patients to other patients. These results suggest that positivity for BAG3 and Mcl-1 may be a key determinant of disease progression in ovarian cancer.

Sensitivity to paclitaxel and expression of BAG3 and Mcl-1 in ovarian cancer cell lines. Earlier study from our group showed that BAG3 knockdown increases the chemosensitivity of ES2 clear ovarian cancer cells, but BAG3 knockdown had no significant effect on the chemosensitivity of AMOC2 serous ovarian cancer cells (28). To clarify the mechanism for this difference, we initially compared the sensitivity to paclitaxel of AMOC2 and ES2 cells using XTT viability assays. When AMOC2 and ES2 cells were treated for $48 \mathrm{~h}$ with increasing concentrations of paclitaxel, we found AMOC2 cells to be significantly more sensitive $(\mathrm{P}<0.0001)$ to the drug than ES2 cells (Fig. 2A). In addition, western blotting showed that BAG3, Mcl-1 and $\mathrm{Bcl}-\mathrm{X}_{\mathrm{L}}$ proteins were all more strongly expressed in ES2 cells than AMOC2 cells (Fig. 2B). Furthermore, we confirmed that silencing Mcl-1increased the chemosensitivity of ES2 cells (Fig. 2C and D). These results suggest that BAG3 may mediate chemoresistance by interacting with anti-apoptotic Bcl2 family proteins.

Overexpression of BAG3 has only a small effect on sensitivity to paclitaxel in AMOC2 serous ovarian cancer cells. We previously showed that in AMOC2 serous ovarian cancer cells, which express Mcl-1 only weakly and are more sensitive to paclitaxel than ES2 cells, BAG3 knockdown has little effect on sensitivity to paclitaxel (28). To further investigate the association between chemoresistance and BAG3 overexpression in vitro, AMOC2 cells were transfected with full-length BAG3. The resultant overexpression of BAG3 led to only slight increases in Mcl-1 expression and paclitaxel resistance, which were not statistically significant (Fig. 2E and F).

Varying miR-29b expression has only a slight effect on sensitivity to paclitaxel in AMOC2 serous ovarian cancer cells. We have also shown that BAG3 knockdown downregulates Mcl-1 expression in ES2 cells through upregulation of microRNA-29b (28). We therefore next investigated the association between BAG3 and miR-29b in AMOC2 cells. As in 
A

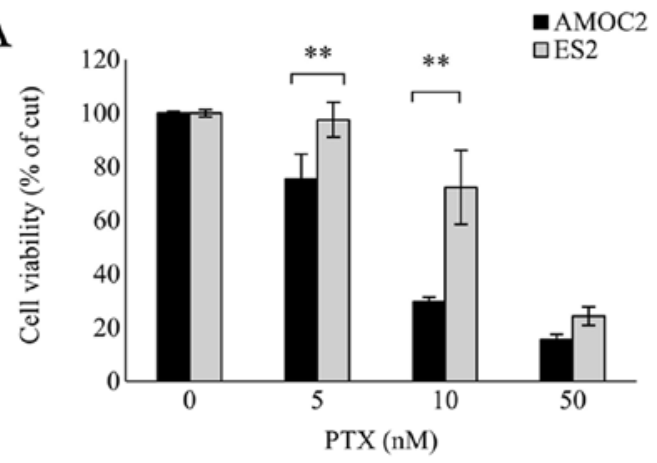

$\mathrm{C}$

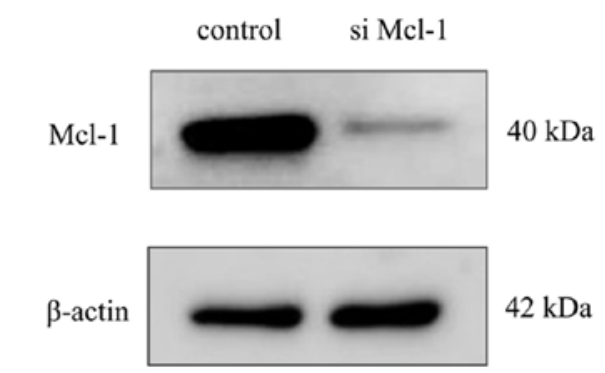

E

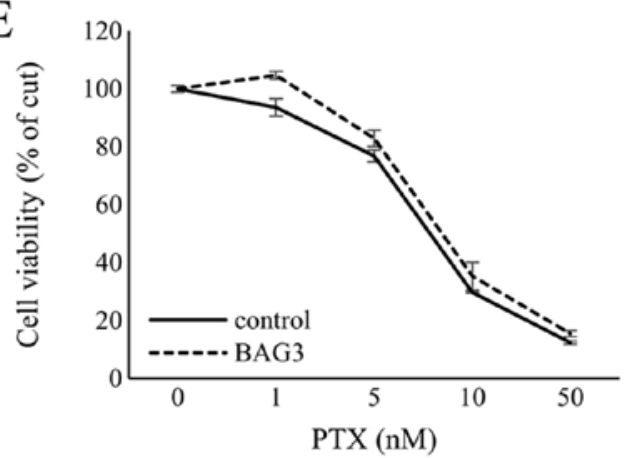

B

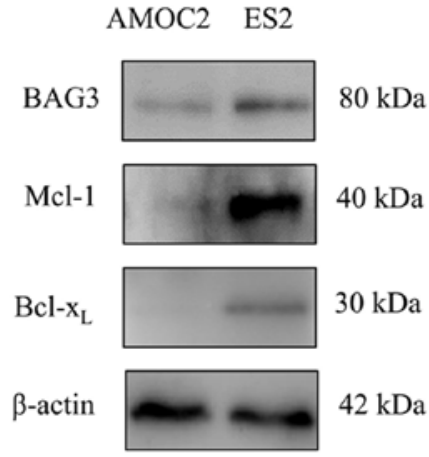

D

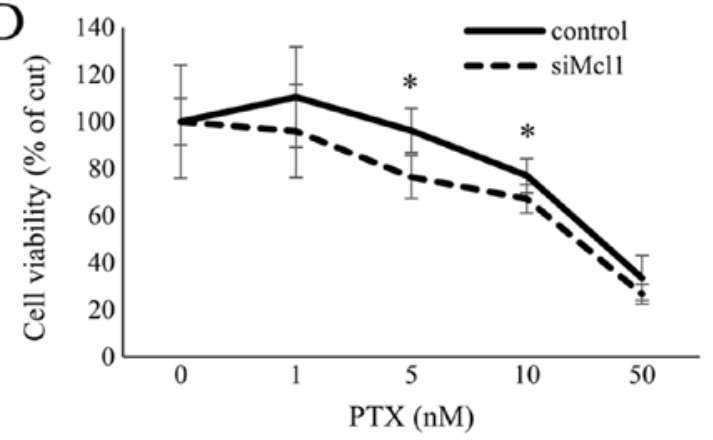

F

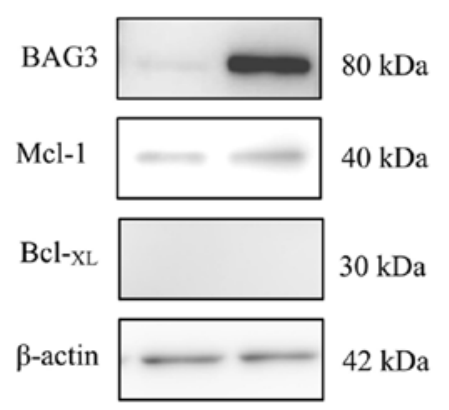

Figure 2. BAG3 and Mcl-1 expression correlates with paclitaxel resistance in ovarian cancer cells. (A) AMOC2 cells and ES2 cells were treated for 48 h with the indicated concentrations of paclitaxel and then subjected to XTT assays. Symbols depict means \pm SD. ${ }^{* *} \mathrm{P}<0.01$. (B) Western blotting showing levels of BAG3, Mcl-1 and Bcl-X $\mathrm{L}_{\mathrm{L}}$ protein in AMOC2 and ES2 cells. As a loading control, the blots were reprobed with anti- $\beta$-actin antibody. (C) Western blot analysis showing Mcl-1 silencing in ES2 cells. As a loading control, the blots were reprobed using mouse monoclonal anti- $\beta$-actin antibody. (D) ES2 cells were transfected with Mcl-1 siRNA (siMcl-1) or non-targeting siRNA (control). Twenty-four hours later paclitaxel was added to a concentration of 0 , 1,5 , 10 or $50 \mathrm{nM}$, and the cells were incubated for an additional $48 \mathrm{~h}$, after which viability was assessed in XTT assays. Shown are means \pm SD; ${ }^{*} \mathrm{P}<0.05$. (E) AMOC2 ovarian cancer cells were transfected with BAG3 expression vector (AMOC2-BAG3) or a control vector (AMOC2-pcDNA). Twenty-four hours later paclitaxel was added to a concentration of $0,1,5,10$ or $50 \mathrm{nM}$, and the cells were incubated for an additional $48 \mathrm{~h}$, after which viability was assessed in XTT assays. (F) Western blot analysis of BAG3, Mcl-1 and Bcl-X $\mathrm{L}_{\mathrm{L}}$ expression in AMOC2-BAG3 and AMOC2-pcDNA cells. As a loading control, the blots were reprobed using mouse monoclonal anti- $\beta$-actin antibody.

ES2 cells, knocking down BAG3 expression using shBAG3 enhanced miR-29b expression in AMOC2 cells (Fig. 3A). In addition, when we transfected AMOC2 cells with miRNA-29b mirVana miRNA mimic to augment miR-29b activity or with miRNA-29b mirVana miRNA inhibitor to diminish miR-29b activity, qRT-PCR confirmed that miR-29b expression was markedly increased in AMOC2 cells transfected with miR29b mimic (Fig. 3B), but decreased in AMOC2 cells transfected with the miR29b inhibitor (Fig. 3C). Unlike in ES2 cells (28), however, transfection of AMOC2 cells with miR-29b-mimic had no effect on Mcl-1 expression or paclitaxel sensitivity, as compared to the miR-control (Fig. 3D and F). On the other hand, Mcl-1 expression was increased somewhat in AMOC2 cells transfected with the miR-29b-inhibitor, and those cells were more refractory to paclitaxel than cells transfected with the miR-29b-inhibitor negative control, though the difference was not statistically significant (Fig. 3E and F). These findings imply that although BAG3 may enhance Mcl-1 expression and chemoresistance in some degree in AMOC2 cells, the effect is mild, inducing little or no endogenous Mcl-1 expression. Nonetheless, these results are consistent with the idea that positivity for both BAG3 and Mcl-1 correlates with a poor prognosis in ovarian cancer patients.

Mcl-1-specific high-affinity antagonist MIM1 increases sensitivity of ES2 cells to paclitaxel. We next used XTT viability assays to assess the effect on cell proliferation of the Mcl-1 antagonist MIM1, alone and in combination with paclitaxel. ES2 cells were treated for $48 \mathrm{~h}$ with increasing concentrations of MIM1 alone or in combination with a low concentration 
A

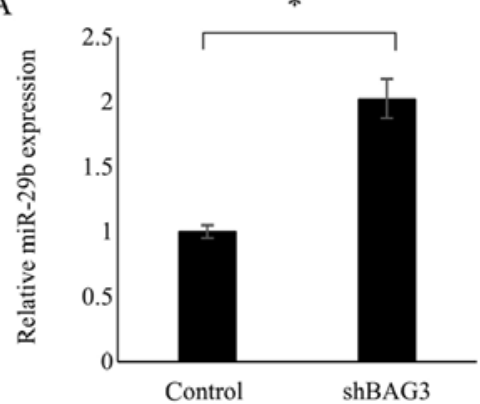

$\mathrm{D}$

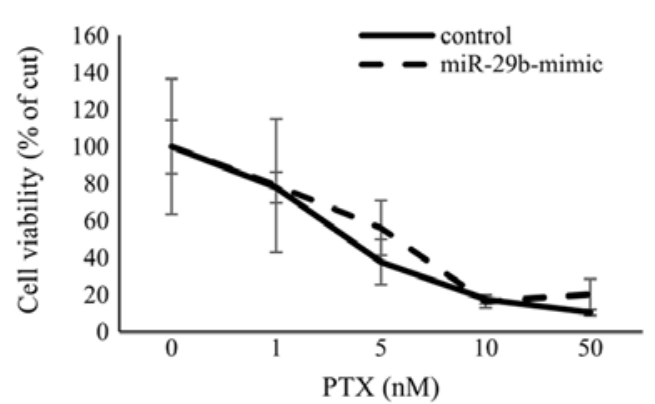

B

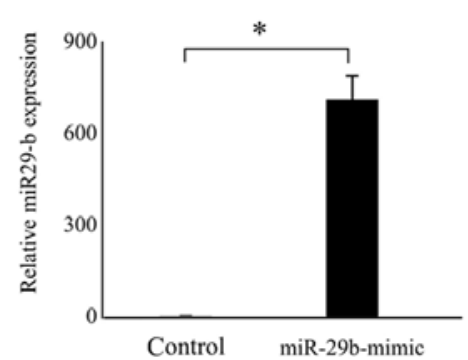

$\mathrm{E}$
$\mathrm{C}$

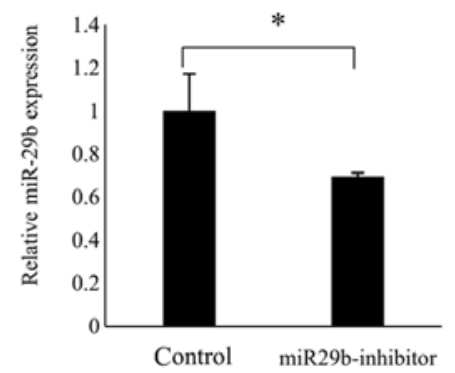

$\mathrm{F}$

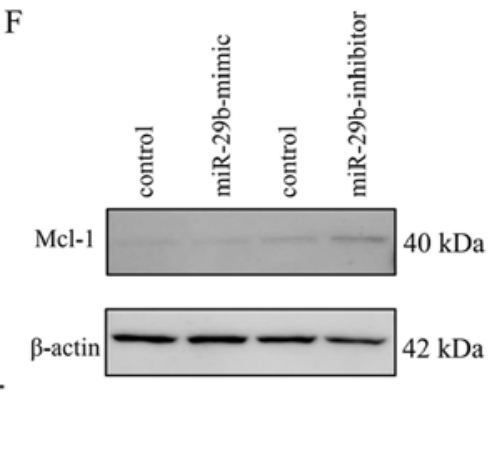

Figure 3. Altering miR-29b expression in AMOC2 cells has little effect on sensitivity to paclitaxel. (A) Real-time qRT-PCR analysis of miR-29b expression in AMOC2 cells infected with a control (LTRH1) or shBAG3 (BAG3 knockdown) viral vector. Bars depict relative microRNA levels normalized to the RNU48 level (internal control). Shown are means $\pm \mathrm{SD}$; $\mathrm{P}<0.05$. (B and C) AMOC2 cells were transfected with miR-29b mirVana miRNA mimic (miR-29b-mimic) to augment miR-29b activity (B) or with miR-29b mirVana inhibitor to diminish miR-29b activity (C). Relative levels of miR-29b expression were then determined using real-time qRT-PCR. Bars depict relative mRNA levels normalized to RNU48 (internal control). Shown are means \pm SD; ${ }^{*} \mathrm{P}<0.05$. (D and E) AMOC2 ovarian cancer cells were transfected with miR-29b-mimic or its control (D), or with a miR-29b-inhibitor or its control (E). Twenty-four hours later paclitaxel was added to a concentration of $0,1,5,10$ or $50 \mathrm{nM}$, and the cells were incubated for an additional $48 \mathrm{~h}$, after which viability was assessed in XTT assays. Symbols depict means \pm SD. (F) AMOC2 cells transfected with miR-29b-mimic or its control, or with miR-29b-inhibitor or its control, were analyzed for Mcl-1 protein expression using western blotting. As a loading control, the blots were reprobed with an anti- $\beta$-actin antibody.
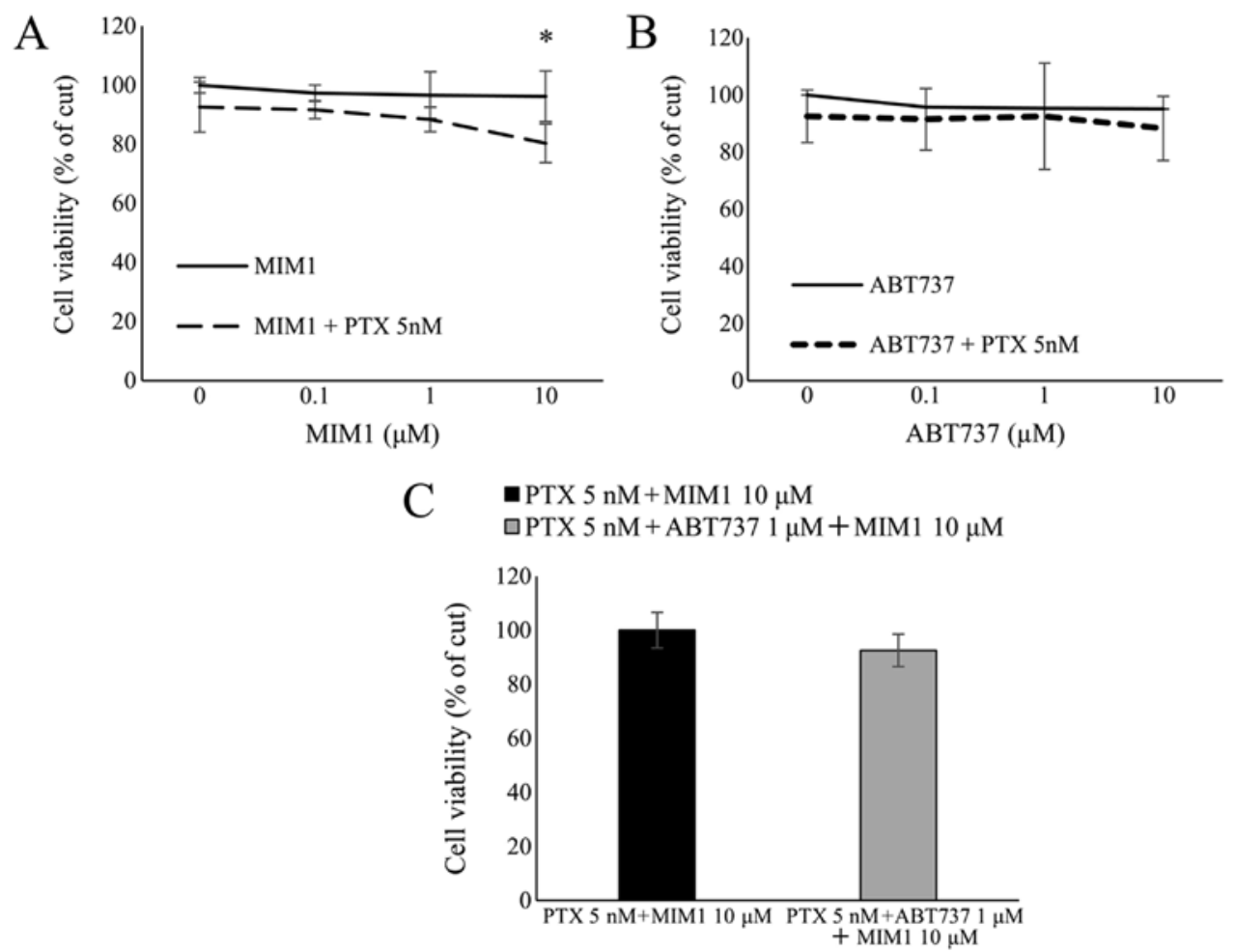

Figure 4. When combined with MIM1, paclitaxel exhibits potent cytotoxic activity against ovarian cancer cells. (A and B) ES2 cells were cultured for $48 \mathrm{~h}$ with the indicated concentrations of MIM1 (A) or ABT737 (B), alone or in combination with $5 \mathrm{nM}$ paclitaxel. Cell viability was then assessed in XTT assays. Symbols depict means $\pm \mathrm{SD} ;{ }^{* *} \mathrm{P}<0.01$. (C) ES2 cells were treated for $48 \mathrm{~h}$ with the combination of $5 \mathrm{nM}$ paclitaxel and $10 \mu \mathrm{M}$ MIM1 or with the combination of $5 \mathrm{nM}$ paclitaxel, $10 \mu \mathrm{M}$ MIM1 and $1 \mu \mathrm{M}$ ABT737. Cell viability was then assessed in XTT assays. Reductions in viability are presented as percentages from the combination of paclitaxel with MIM1. Symbols depict means \pm SD. 
A

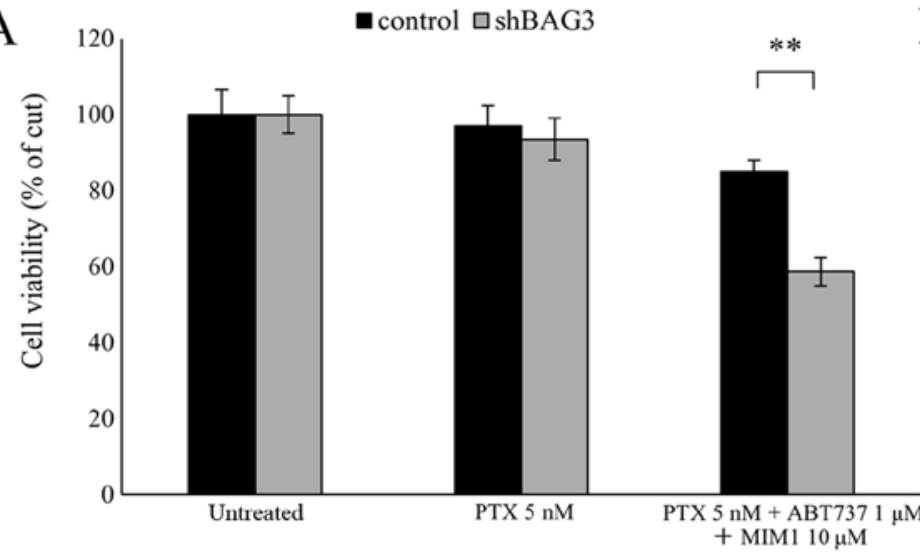

B

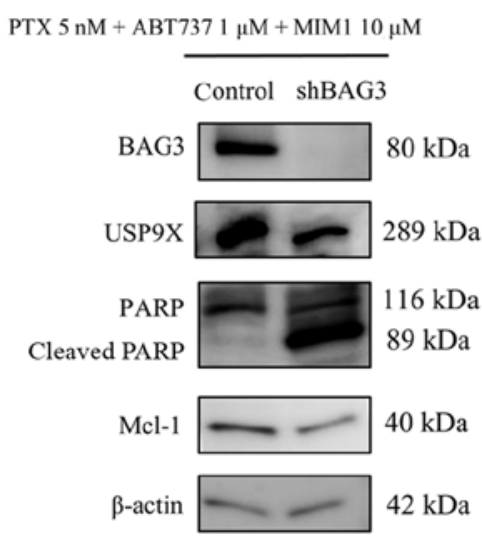

$\mathrm{C}$

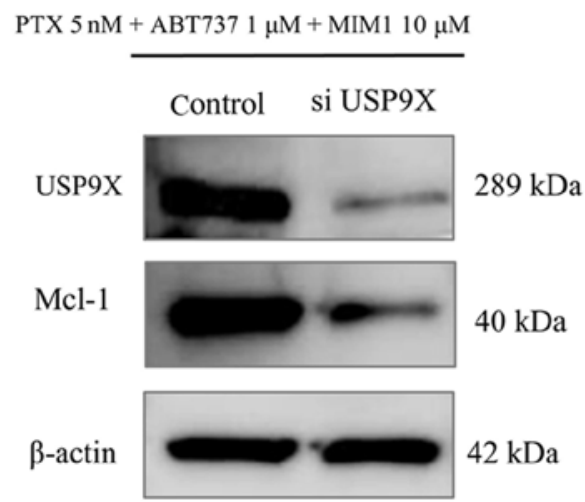

$\mathrm{D}$

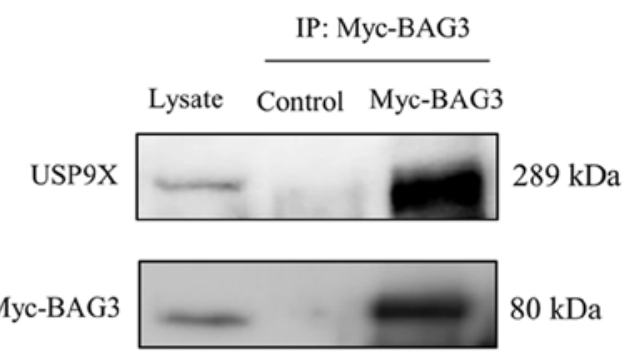

Figure 5. BAG3 regulates Mcl-1 levels through interaction with USP9X and is related to chemoresistance. (A) ES2 BAG3 knockdown (ES2-shBAG3) cells and their controls (ES2-LTRH1) were cultured for $48 \mathrm{~h}$ with $5 \mathrm{nM}$ paclitaxel alone or a combination with $1 \mu \mathrm{M}$ ABT737 and $10 \mu \mathrm{M}$ MIM1. Cell viability was then assessed in XTT assays. Symbols depict means \pm SD. ${ }^{* *} \mathrm{P}<0.01$. (B) ES2-shBAG3 and ES2-LTRH1 cells were treated for $48 \mathrm{~h}$ with a combination of $5 \mathrm{nM}$ paclitaxel, $1 \mu \mathrm{M}$ ABT737 and $10 \mu \mathrm{M}$ MIM-1, after which the cells were subjected to western blot analysis for BAG3, USP9X, cleaved PARP and Mcl-1. As a loading control, the blots were reprobed using anti- $\beta$-actin antibody. (C) ES2 cells were transfected with USP9X siRNA (si USP9X) or non-targeting siRNA (control). Twenty-four hours later $5 \mathrm{nM}$ paclitaxel, $1 \mu \mathrm{M} \mathrm{ABT737}$ and $10 \mu \mathrm{M}$ MIM-1 were added, and the cells were incubated for an additional $48 \mathrm{~h}$, after which the cells were subjected to western blot analysis for USP9X and Mcl-1. As a loading control, the blots were reprobed using mouse monoclonal anti$\beta$-actin antibody. (D) Co-immunoprecipitation and western blotting revealed the interaction between BAG3 and USP9X in ES2 cells. Total cell lysates were collected from ES2 cells expressing empty pcDNA vector (control) or Myc-BAG3. Samples were immunoprecipitated using an anti-Myc antibody and then blotted with anti-USP9X antibody and anti-Myc antibody (right lane). The middle lane was blotted with control. The left lane contained normal ES2 lysates and also showed the presence of USP9X and Myc-BAG3.

of paclitaxel (5 $\mathrm{nM})$. Treatment with the paclitaxel/MIM1 combination significantly reduced cell viability, whereas MIM1 had no effect on cell viability by itself (Fig. 4A). Thus MIM1 appears to increase the sensitivity of Mcl-1-expressing cells to paclitaxel. We also examined the effect of combining paclitaxel with $\mathrm{ABT} 737$, a Bcl-2, $\mathrm{Bcl}-\mathrm{X}_{\mathrm{L}}$ and $\mathrm{Bcl}_{-\mathrm{w}}$ antagonist, in a similar manner. We found that ABT737 alone or in combination with paclitaxel had little effect on ES2 cell viability (Fig. 4B), which is consistent with earlier reports showing that Mcl-1 mediates ABT737 resistance $(11,22,29,30)$.

BAG3 knockdown enhances the effect of the triple-drug combination of paclitaxel, MIMI and ABT737. We also evaluated the effect of combining paclitaxel $(5 \mathrm{nM})$, MIM1 $(10 \mu \mathrm{M})$ and ABT737 $(1 \mu \mathrm{M})$ and found that the effect of the triple-drug combination on cell viability was not different from that of paclitaxel and MIM1 (Fig. 4C). We speculated that, as suggested previously, BAG3 stabilizes and upregulates Mcl-1 $(22,28)$ and that as a consequence the inhibitory effect of MIM1 is not sufficient to increase the efficacy of ABT737. To test this idea, ES2 cells were infected with a retroviral vector encoding shRNA targeting BAG3 (ES2-shBAG3). As expected, BAG3 knockdown significantly enhanced the effect of the triple-drug combination on cell viability (Fig. 5A). In ES2-shBAG3 cells treated with the triple-drug combination, Mcl-1 expression was reduced, as compared to control, and PARP cleavage, an indicator of caspase activation, were observed (Fig. 5B). These results suggest that stabilization of Mcl-1 by BAG3 both confers resistance to chemotherapy and contributes to the anti-apoptotic activity within ovarian cancer cells.

Interaction between BAG3 and USP9X. Finally, we tested whether levels of the deubiquitinase USP9X, which stabilizes Mcl-1 by negatively regulating its ubiquitination, are affected by BAG3. We initially found that USP9X levels are reduced by BAG3 knockdown (Fig. 5B) and Mcl-1 levels are decreased by silencing USP9X (Fig. 5C). Then to determine whether BAG3 directly interacts with USP9X, we transfected a Myc-tagged BAG3 expression construct into ES2 cells. Thereafter,immunoprecipitation of the Myc epitope from lysates of the Myc-BAG3 transfectants also pulled down endogenous USP9X, whereas 


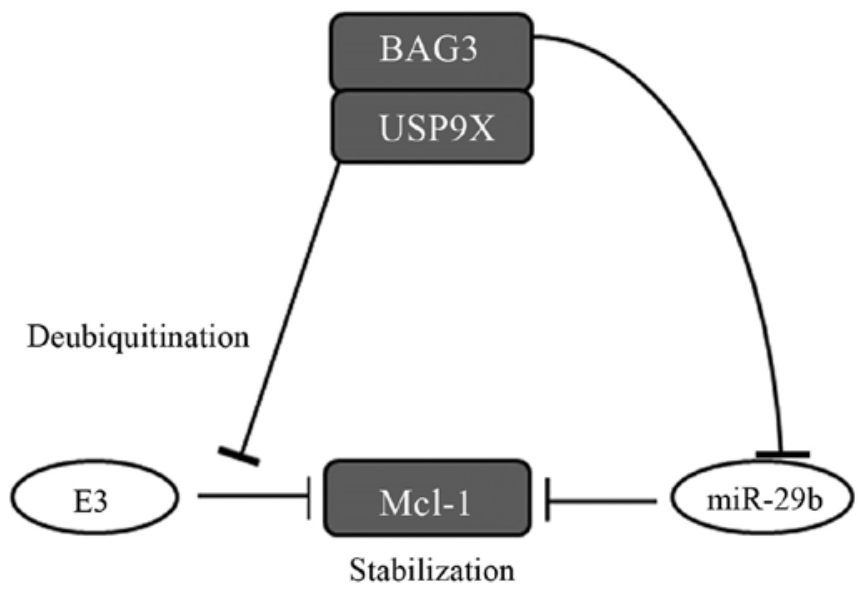

Figure 6. BAG3 stabilizes Mcl-1 expression in ovarian cancer cells. Role for BAG3 in Mcl-1 stabilization and cancer cell chemoresistance. BAG3 stabilizes Mcl-1 through its interaction with USP9X and by reducing miR-29b expression.

neither protein was precipitated from control lysates (Fig. 5D). Apparently, BAG3 and USP9X directly interact within ES2 ovarian cancer cells. Taken together, these results suggest that BAG3 binds to and stabilizes USP9X, which in turn stabilizes Mcl-1 and promotes resistance to apoptosis.

\section{Discussion}

In this study, we investigated the relationship between the combined expression of BAG3 and Mcl-1 in ovarian cancer tissue and patient prognosis and chemoresistance. We previously showed that tumoral BAG3 expression was associated with a significantly increased risk of disease progression and recurrence (26). Our present analysis indicates that Mcl-1 expression in primary ovarian cancer tissues is associated with a poor prognosis and that expression of both BAG3 and Mcl-1 is associated with a significantly poorer prognosis than expression of BAG3 alone. These findings suggest that tumoral BAG3 does not act alone in contributing to a poor prognosis in ovarian cancer patients, but acts in combination with Mcl-1. In vitro, ES2 clear ovarian cancer cells endogenously express high levels of Mcl-1 and BAG3, and show less sensitivity to paclitaxel than AMOC2 serous ovarian cancer cells, which endogenously express only low levels of Mcl-1 and BAG3. BAG3 knockdown in ES2 cells suppresses Mcl-1 expression and increases sensitivity to paclitaxel (28), which is consistent with the idea that combined expression of both BAG3 and Mcl-1 correlates with a poor prognosis in ovarian cancer patients, and that BAG3 and Mcl-1 act in concert to mediate anti-apoptotic activity in ovarian cancer cells.

In many cancers, Mcl-1 is a key mediator that enables cancer cells to overcome oncogenic stress-induced apoptosis. For instance, Mcl-1 is critical for the development and maintenance of acute myeloid leukemia $(31,32)$, and high levels of Mcl-1 are often associated with chemotherapeutic resistance and relapse $(33,34)$. Moreover, Mcl-1 is not inhibited by the Bcl-2 antagonist ABT737 and is considered responsible for cancer cell resistance to ABT737 $(11,22,29,30)$. Consistent with those earlier findings, our results show that ABT737 does not increase paclitaxel sensitivity in Mcl-1-expressing ES2 cells. On the other hand, MIM1, a selectively Mcl-1 inhibitor (13), increased somewhat the sensitivity of ES2 cells to paclitaxel. However, adding ABT737 to the paclitaxel/MIM1 combination provided no additional benefit. A large body of evidence, including our earlier research, indicates that BAG3 induces chemoresistance through upregulation of Mcl-1 expression $(22,23,28)$. We therefore hypothesized that in the presence of BAG3, the inhibitory effect of MIM1 would not be sufficient to suppress Mcl-1 activity, and thus ABT737 would also be without effect. Indeed, we found that BAG3 knockdown (ES2-shBAG3) using a retroviral shRNA vector increased the sensitivity of ES2 cells to the combination of paclitaxel, MIM1 and ABT737. Several studies have investigated the mechanism by which BAG3 positively regulates Mcl-1 expression. Using a colon cancer cell line, Jacobs and Marnett demonstrated that heat shock factor 1 (HSF1) upregulates Mcl-1 expression and that the effect is mediated by BAG3 (23). In addition, it is now known that BAG3 binds to and stabilizes Mcl-1 (22), and that BAG3 also upregulates Mcl-1 expression by inhibiting miR-29b expression (28). Here we provide the first evidence of yet another molecular mechanism by which BAG3 upregulates Mcl-1 expression.

It is now known that four different E3 ubiquitin-ligases (Mule, SCF $\beta$-TrCP, SCFFbw7 and Trim17) (35-38) mediate Mcl-1 ubiquitination, while the deubiquitinase USP9X acts to suppress Mcl-1 ubiquitination (24). We found that BAG3 binds to and stabilizes USP9X and that USP9X expression is downregulated during apoptosis in ES2 cells after BAG3 knockdown. In addition, our results show that downregulation of Mcl-1 expression correlates with attenuated USP9X expression, suggesting BAG3 upregulates Mcl-1 levels via USP9X. A schematic diagram showing a possible mechanism to explain the BAG3 activity is shown in Fig. 6. Notably, BAG1, another BAG family protein, also reportedly binds to USP9X and regulates the stability of Mcl-1 (39). Both BAG1 and BAG3 contain conserved BAG domains via which they bind Hsp70 $(14,15)$, interact with the proteasome and modulate Hsp70 client protein degradation $(40,41)$. We therefore suggest that BAG3 also interacts with USP9X and that the stability of USP9X is determined to both BAG3 and BAG1.

In conclusion, we have shown that the combined expression of BAG3 and Mcl-1 correlates with a poor prognosis in ovarian cancer patients and with resistance to paclitaxel. Mcl-1 inhibition by MIM1 effectively increased paclitaxel sensitivity, overcoming the chemoresistance of ovarian cancer cells. Addition of the Bcl-2 antagonist ABT737 increased paclitaxel sensitivity further, but only after BAG3 knockdown. We suggest BAG3 binds to USP9X to stabilize Mcl-1 levels, and that BAG3 and Mcl-1 are potentially useful biomarkers of the responsiveness of ovarian cancer to paclitaxel. Moreover, BAG3 would likely be a useful therapeutic target, particularly if targeted in combination with Bcl-2 family proteins, for the treatment of chemoresistant ovarian cancers.

\section{Acknowledgements}

The authors would like to thank Dr S. Takayama for providing a gene silencing vector (pLTRH1) specific for BAG3 and rabbit anti-BAG3 antibody. 


\section{References}

1. McGuire WP, Hoskins WJ, Brady MF, Kucera PR, Partridge EE, Look KY, Clarke-Pearson DL and Davidson M: Cyclophosphamide and cisplatin compared with paclitaxel and cisplatin in patients with stage III and stage IV ovarian cancer N Engl J Med 334: 1-6, 1996.

2. Itamochi H, Kigawa J and Terakawa N: Mechanisms of chemoresistance and poor prognosis in ovarian clear cell carcinoma. Cancer Sci 99: 653-658, 2008.

3. Anglesio MS, Carey MS, Köbel M, Mackay H and Huntsman DG; Vancouver Ovarian Clear Cell Symposium Speakers: Clear cell carcinoma of the ovary: A report from the first Ovarian Clear Cell Symposium, June 24th, 2010. Gynecol Oncol 121: 407-415, 2011.

4. Hanahan D and Weinberg RA: The hallmarks of cancer. Cell 100: 57-70, 2000.

5. Johnstone RW, Ruefli AA and Lowe SW: Apoptosis: A link between cancer genetics and chemotherapy. Cell 108: 153-164, 2002.

6. Cory S, Huang DCS and Adams JM: The Bcl-2 family: Roles in cell survival and oncogenesis. Oncogene 22: 8590-8607, 2003.

7. Yip KW and Reed JC: Bcl-2 family proteins and cancer. Oncogene 27: 6398-6406, 2008

8. Czabotar PE, Lessene G, Strasser A and Adams JM: Control of apoptosis by the BCL-2 protein family: Implications for physiology and therapy. Nat Rev Mol Cell Biol 15: 49-63, 2014.

9. Llambi F, Moldoveanu T, Tait SW, Bouchier-Hayes L, Temirov J, McCormick LL, Dillon CP and Green DR: A unified model of mammalian BCL-2 protein family interactions at the mitochondria. Mol Cell 44: 517-531, 2011

10. Oltersdorf T, Elmore SW, Shoemaker AR, Armstrong RC, Augeri DJ, Belli BA, Bruncko M, Deckwerth TL, Dinges J, Hajduk PJ, et al: An inhibitor of Bcl-2 family proteins induces regression of solid tumours. Nature 435: 677-681, 2005.

11. van Delft MF, Wei AH, Mason KD, Vandenberg CJ, Chen L, Czabotar PE, Willis SN, Scott CL, Day CL, Cory S, et al: The BH3 mimetic ABT-737 targets selective Bcl-2 proteins and efficiently induces apoptosis via Bak/Bax if Mcl-1 is neutralized. Cancer Cell 10: 389-399, 2006.

12. Wertz IE, Kusam S, Lam C, Okamoto T, Sandoval W, Anderson DJ, Helgason E, Ernst JA, Eby M, Liu J, et al: Sensitivity to antitubulin chemotherapeutics is regulated by MCL1 and FBW7. Nature 471: 110-114, 2011.

13. Cohen NA, Stewart ML, Gavathiotis E, Tepper JL, Bruekner SR, Koss B, Opferman JT and Walensky LD: A competitive stapled peptide screen identifies a selective small molecule that overcomes MCL-1-dependent leukemia cell survival. Chem Biol 19: $1175-1186,2012$

14. Takayama S, Xie Z and Reed JC: An evolutionarily conserved family of Hsp70/Hsc70 molecular chaperone regulators. J Biol Chem 274: 781-786, 1999.

15. Rosati A, Ammirante M, Gentilella A, Basile A, Festa M, Pascale M, Marzullo L, Belisario MA, Tosco A, Franceschelli S, et al: Apoptosis inhibition in cancer cells: A novel molecular pathway that involves BAG3 protein. Int J Biochem Cell Biol 39: $1337-1342,2007$

16. Einbond A and Sudol M: Towards prediction of cognate complexes between the WW domain and proline-rich ligands. FEBS Lett 384: 1-8, 1996.

17. Sudol M, Chen HI, Bougeret C, Einbond A and Bork P: Characterization of a novel protein-binding module - the WW domain. FEBS Lett 369: 67-71, 1995.

18. Iwasaki M, Homma S, Hishiya A, Dolezal SJ, Reed JC and Takayama S: BAG3 regulates motility and adhesion of epithelial cancer cells. Cancer Res 67: 10252-10259, 2007.

19. Rosati A, Bersani S, Tavano F, Dalla Pozza E, De Marco M, Palmieri M, De Laurenzi V, Franco R, Scognamiglio G, Palaia R, et al: Expression of the antiapoptotic protein BAG3 is a feature of pancreatic adenocarcinoma and its overexpression is associated with poorer survival. Am J Pathol 181: 1524-1529, 2012.

20. Festa M, Del Valle L, Khalili K, Franco R, Scognamiglio G, Graziano V, De Laurenzi V, Turco MC and Rosati A: BAG3 protein is overexpressed in human glioblastoma and is a potential target for therapy. Am J Pathol 178: 2504-2512, 2011.

21. Chiappetta G, Basile A, Barbieri A, Falco A, Rosati A, Festa M, Pasquinelli R, Califano D, Palma G, Costanzo R, et al: The anti-apoptotic BAG3 protein is expressed in lung carcinomas and regulates small cell lung carcinoma (SCLC) tumor growth. Oncotarget 5: 6846-6853, 2014.
22. Boiani M, Daniel C, Liu X, Hogarty MD and Marnett LJ: The stress protein BAG3 stabilizes Mcl-1 protein and promotes survival of cancer cells and resistance to antagonist ABT-737. J Biol Chem 288: 6980-6990, 2013.

23. Jacobs AT and Marnett LJ: HSF1-mediated BAG3 expression attenuates apoptosis in 4-hydroxynonenal-treated colon cancer cells via stabilization of anti-apoptotic $\mathrm{Bcl}-2$ proteins. J Biol Chem 284: 9176-9183, 2009.

24. Schwickart M, Huang X, Lill JR, Liu J, Ferrando R, French DM, Maecker H, O'Rourke K, Bazan F, Eastham-Anderson J, et al: Deubiquitinase USP9X stabilizes MCL1 and promotes tumour cell survival. Nature 463: 103-107, 2010.

25. Yan J, Zhong N, Liu G, Chen K, Liu X, Su L and Singhal S: Usp9x- and Noxa-mediated Mcl-1 downregulation contributes to pemetrexed-induced apoptosis in human non-small-cell lung cancer cells. Cell Death Dis 5: e1316, 2014.

26. Suzuki M, Iwasaki M, Sugio A, Hishiya A, Tanaka R, Endo T, Takayama S and Saito T: BAG3 (BCL2-associated athanogene 3) interacts with MMP-2 to positively regulate invasion by ovarian carcinoma cells. Cancer Lett 303: 65-71, 2011.

27. Homma S, Iwasaki M, Shelton GD, Engvall E, Reed JC and Takayama S: BAG3 deficiency results in fulminant myopathy and early lethality. Am J Pathol 169: 761-773, 2006.

28. Sugio A, Iwasaki M, Habata S, Mariya T, Suzuki M, Osogami H, Tamate M, Tanaka R and Saito T: BAG3 upregulates Mcl-1 through downregulation of miR-29b to induce anticancer drug resistance in ovarian cancer. Gynecol Oncol 134: 615-623, 2014.

29. Konopleva M, Contractor R, Tsao T, Samudio I, Ruvolo PP, Kitada S, Deng X, Zhai D, Shi YX, Sneed T, et al: Mechanisms of apoptosis sensitivity and resistance to the BH3 mimetic ABT-737 in acute myeloid leukemia. Cancer Cell 10: 375-388, 2006.

30. Tahir SK, Yang X, Anderson MG, Morgan-Lappe SE, Sarthy AV, Chen J, Warner RB, Ng SC, Fesik SW, Elmore SW, et al: Influence of Bcl-2 family members on the cellular response of small-cell lung cancer cell lines to ABT-737. Cancer Res 67: 1176-1183, 2007.

31. Glaser SP, Lee EF, Trounson E, Bouillet P, Wei A, Fairlie WD, Izon DJ, Zuber J, Rappaport AR, Herold MJ, et al: Mechanisms of chemoresistance and poor prognosis in ovarian clear cell carcinoma. Genes Dev 26: 120-125, 2012

32. Xiang Z, Luo H, Payton JE, Cain J, Ley TJ, Opferman JT and Tomasson MH: Mcll haploinsufficiency protects mice from Myc-induced acute myeloid leukemia. J Clin Invest 120: 2109-2118, 2010

33. Wei G, Twomey D, Lamb J, Schlis K, Agarwal J, Stam RW, Opferman JT, Sallan SE, den Boer ML, Pieters R, et al: Gene expression-based chemical genomics identifies rapamycin as a modulator of MCL1 and glucocorticoid resistance. Cancer Cell 10: 331-342, 2006

34. Wuillème-Toumi S, Robillard N, Gomez P, Moreau P, Le Gouill S, Avet-Loiseau H, Harousseau JL, Amiot M and Bataille R: Mcl-1 is overexpressed in multiple myeloma and associated with relapse and shorter survival. Leukemia 19: 1248-1252, 2005.

35. Zhong Q, Gao W, Du F and Wang X: Mule/ARF-BP1, a BH3-only E3 ubiquitin ligase, catalyzes the polyubiquitination of Mcl-1 and regulates apoptosis. Cell 121: 1085-1095, 2005.

36. Ding Q, He X, Hsu JM, Xia W, Chen CT, Li LY, Lee DF, Liu JC, Zhong Q, Wang X, et al: Degradation of Mcl-1 by beta-TrCP mediates glycogen synthase kinase 3-induced tumor suppression and chemosensitization. Mol Cell Biol 27: 4006-4017, 2007.

37. Inuzuka H, Shaik S, Onoyama I, Gao D, Tseng A, Maser RS, Zhai B, Wan L, Gutierrez A, Lau AW, et al: SCF(FBW7) regulates cellular apoptosis by targeting MCL1 for ubiquitylation and destruction. Nature 471: 104-109, 2011

38. Magiera MM, Mora S, Mojsa B, Robbins I, Lassot I and Desagher S: Trim17-mediated ubiquitination and degradation of Mcl-1 initiate apoptosis in neurons. Cell Death Differ 20: 281-292, 2013.

39. Aveic S, Pigazzi M and Basso G: BAG1: The guardian of antiapoptotic proteins in acute myeloid leukemia. PLoS One 6: e26097, 2011.

40. Lüders J, Demand J and Höhfeld J: The ubiquitin-related BAG-1 provides a link between the molecular chaperones Hsc70/Hsp70 and the proteasome. J Biol Chem 275: 4613-4617, 2000.

41. Doong H, Rizzo K, Fang S, Kulpa V, Weissman AM and Kohn EC: CAIR-1/BAG-3 abrogates heat shock protein-70 chaperone complex-mediated protein degradation: Accumulation of poly-ubiquitinated Hsp90 client proteins. J Biol Chem 278: 28490-28500, 2003. 